\title{
Die prädikative Funktion des Verbalnomens auf *pa im Juraksamojedischen
}

In meiner Arbeit "Zur juraksamojedischen Konjugation» habe ich mich bereits mit der prädikativen Funktion der possessivsuffigierten Formen des jurakischen Verbalnomens befasst und festgestellt, dass diese Funktion dem prädikativen Gebrauch der ungarischen Verbaladverbien auf va/ve entspricht, den wir aus den ältesten ungarischen Kodexen kennen (FUF XXXVIII 176-179). Die Ableitungssuffixe des ungarischen und des jurakischen Verbalnomens gehören bekanntlich zusammen, und ebendeshalb habe ich es für nötig erachtet, die syntaktische Funktion des jurakischen Verbalnomens zusätzlich zu untersuchen, nicht nur im Zusammenhang mit den damit enger verbundenen Satzgliedern, sondern auch im Rahmen des ganzen Satzes. In meinem oben erwähnten Aufsatz habe ich mich auf eine Studie von Sándor Károly berufen, ${ }^{1}$ in der uns reichliches ungarisches Material in gründlicher Bearbeitung zur Verfügung steht. Der Vergleich jurakischer und ungarischer sprachlicher Tatsachen hat also eine feste Grundlage. Es ist zu beachten, dass der prädikative Gebrauch der Verbaladverbien auf va/ve eine charakteristisch ungarische Eigentümlichkeit ist; der ursprüngliche lateinische Text der Kodexe hat keinerlei Anlass dazu gegeben (vgl. Károly, a.a.0. 213).

Die Bedeutung der Grundform des jurakischen Verbalnomens entspricht im allgemeinen der Bedeutung der ungarischen "Aktionsnomina mit dem Suffix ás/és" (vgl. Károly, ebd. 224), in manchen Fällen steht sie der Bedeutung der ungari-

\footnotetext{
${ }^{1}$ Igenévrendszerünk a kódexirodalom első szakaszában (Das System der ungarischen Verbalnomina in der ersten Periode der Kodexliteratur + Zusammenfassung), Budapest 1956.
} 
schen Hauptwörter vom Typ élet 'Leben' bzw. hazugság 'Lüge' näher. Das jurakische Verbalnomen erscheint in den Texten mit der Endung $b a, B a$ bzw. - besonders in intervokalischer Position - mit $w a, \beta \beta a .^{1}$ Die Grundform des Verbalnomens wird in prädikativer Funktion am häufigsten in Verbindung mit einem Subjekt in der 2.P.Sg. gebraucht; sie bekommt in dieser Funktion a u $\mathrm{n}$ a h m slos das Possessivsuffix $*_{t}{ }^{2}$ Sämtliche hierher gehörige Belege sind Zitate aus Gesprächen von Personen: (C) tjukaeda häe-wa-t | siu jirī | pinjijū mindān 'wenn du von hier fortgehst, | sieben Monate | weiter weg gehst' (35); vgl. Variante b. häe-ba (49), c. häe-ba-ta (56). In der Bedeutung des Verbalnomens mit und ohne Personalsuffix besteht - ähnlich wie im ungarischen Sprachgebrauch (vgl. Károly, ebd. 209) - kein Unterschied. Sätze, die auch ein mit * pa gebildetes Verbalnomen als Prädikat enthalten, bestehen in Ị̇beinstinmung mit der Konstruktion des oben zitierten Satzes aus zwei Verbindungen: aus der Verbindung von einem durch ein Verbalnomen auf ${ }^{*} p a$ ausgedrückten Prädikat (im folgenden verbalnominales Prädikat) und seinen Ergänzungen, sowie aus der Verbindung von einem Verbalprädikat und seinen Ergänzungen. Das Verbalprädikat ist im wesentlichen ebenfalls

${ }^{1}$ Nach vereinzelten Angaben kommt die Grundform des Verbalnomens mit und ohne Personalsuffix auch als Subjekt vor: (C) sieusva waewo; talewa waewo 'das Lügen ist schlecht; das Stehlen ist schlecht' (Anh 376); meadm miwa tārā; tym teamdawa tārā 'man muss das Zelt aufrichten; man muss das Rentier kaufen' (ebd.); neäkollabat ( neäkollaba) waewau? 'ist das schlimm, wenn du das Netz ziehst?' (128). (In einem Beleg jedoch BS tāapā-r 'dein Geben' 577.)

2 Nach Castréns Grammatik erscheint das Possessivsuffix der 2.P.Sg. $*_{t}$. in der possessiven Deklination nur in Formen mit Kasussuffixen. Aus den Texten können wir aber vereinzelte Belege zitieren, um zu beweisen, dass das Px. ${ }^{*} t$ ursprünglich auch mit der Grundform der

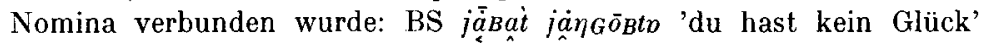

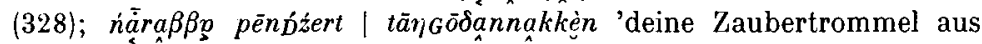
Kupfer |'gibst du wohl' (331); MS pinnne $\beta \approx$ ènnéekkōrt jängu'draussen ist nicht einmal ein Hund' (305). In Verwandtschaftsnamen, ferner auch als determinierendes Element tritt das Px. ${ }^{*} \cdot$ auf: (C) ninjedaet jāna 'im Lande des Mannes deiner älteren Schwester' (245); siuddubeät njuh 'der Sohn des Riesen' (176); Kan niśśet $\mid$ tī $\delta p \bar{o} k k p \underline{p}$ 'dein Vater hat viel Rentiere' (239) usw. 
ein Verbalnomen, es nimmt aber die in der Konjugation gebrauchten Personalsuffixe an. Das Verbalprädikat drückt das Hauptgeschehen aus, das verbalnominale Prädikat aber ein solches "untergeordnetes Geschehen", von dem die Verwirklichung des Hauptgeschehens abhängt: es bezeichnet also die Umstände des Eintreffens des Hauptgeschehens, seine Bedingung, seine Ursache, Art und Weise, Zeit usw. Alle diese Bedeutungen können - wie auch im Ungarischen - aus der Grundbedeutung der Zustandsbestimmung abgeleitet werden, sie sind auch nicht deutlich voneinander getrennt (vgl. Károly, ebd. 156-160). Da das jurakische Verbalprädikat kein konkretes Geschehen ausdrückt, sondern nur Dinge, die in der $\mathrm{Zu}$ kunft eintreffen können, also unbestimmt sind, ist es von jurakischem Gesichtspunkt aus nicht überraschend, dass am verbalen wie am verbalnominalen Prädikat Verbalnomensuffixe erscheinen können, die in gewissem Sinne eine Irrealität bezeichnen (vgl. FUF XXXVIII 153). Um Wiederholıngen zu vermeiden bemerke ich gleich hier, dass ein verbalnominales oder ein verbates Prädikat, das aus einem intransitiven Verb gebildet ist, nur eine adverbiale Bestimmung haben kann, Prädikate aber, die aus transitivem Verb oder aus intransitivem Verb mit transitiver Konjugation stammen, können auch bezeichnete und unbezeichnete Objekte haben. Der Name des Objekts steht unmittelbar vor dem Prädikat (nur ein eng zum Prädikat gehörendes Gerundium oder ein Verbalnomen mit Lativsuffix kann davor stehen). Das verbalnominale Prädikat mit Ergänzungen steht - mit seltenen Ausnahmen - vor dem Verbalprädikat bzw, vor der Verbindung des Verbalprädikats und seiner Ergänzungen. ${ }^{1}$

In den folgenden Sätzen haben, wie auch in dem obigen Beispielsatz, sowohl das verbalnominale als auch das verbale Prädikat ein gemeinsames Subjekt in der 2.P.Sg. Das Subjekt wird selten genannt, auf seine Person weist das Personalsuffix an den Prädikaten hin: (C) niewemda seämbad | hartauan sauan(a) 'wenn du deine Mutter bedauerst, | bist du ja selbst

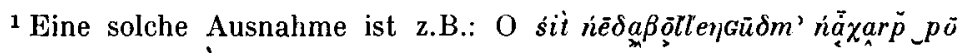
jillte $\beta \beta \bar{a} n^{\prime} \chi_{\wedge}^{a r \beta} \bar{a} \dot{\beta} \bar{a}_{B} \alpha t$ 'ich dinge dich, wenn du drei Jahre leben willst' (39). 
gut' (284); haroabat tōndajir 'willst du, so magst du bedecken'

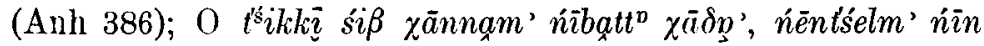
$m$ sèt ' 'wenn du diese sieben Blutopfer nicht tötest, geht es dir'

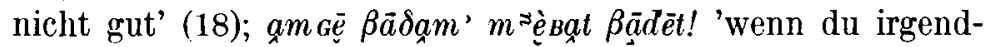

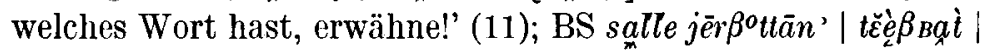
tikkī nọi pannemt śêránd! 'bei deiner Ankunft | bei dem Fürsten von Obdorsk | jenen deinen Tuchrock / lege an!' (366). - In einigen Fällen drückt ein verbalnominales Prädikat auch das

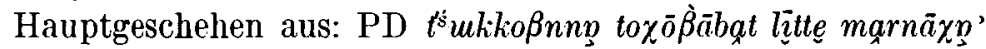

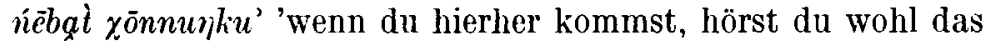
Knistern deiner Knochen nicht' (Mat 415). - Im Satz wird die Verbindung des verbalen und des verbalnominalen Prädikats (und ihrer Ergänzungen) lockerer, das Abhängigkeitsverhältnis zwischen dem Hauptgeschehen und dem mit verbalnominalem Prädikat ausgedrückten "untergeordneten Geschehen" erscheint in deutlicherer Form, wenn die zweierlei Prädikate kein gemeinsames Subjekt haben: (C) pudar sïjebat lādanudm 'wenn du lügst, werde ich dich schlagen' (Anh 379); (L) O pūnny

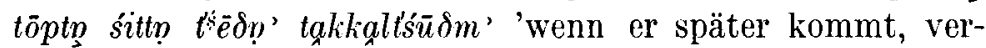

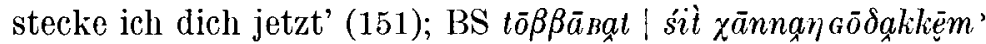
'wenn du kommst, I nehme ich dich wohl mit' (575); (C) pudar

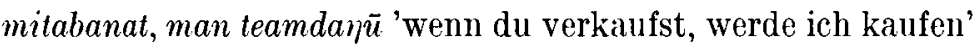
(Anh 385). Die durch das verbalnominale Prädikat ausgedrückte Bedingung erhält Nachdruck durch das Ableitungssuffix des Opt.-Kond.-Pot. na (vgl. Károly, ebd. 208-213, 231-236). - Passivsuffixe geben den Prädikaten passivischen

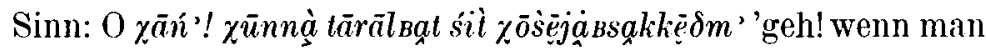
dich irgendwann braucht, werde ich dich wohl holen kommen'

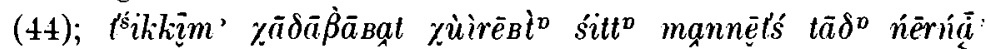

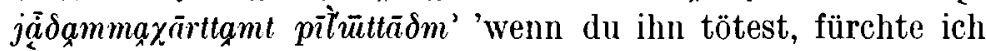
mich nicht nur, wenn ich dich sehe, sondern von da an werde ich auch deine Spuren fürchten' (50). - Das Subjekt in der 3. Person beim verbalen Prädikat ist genannt: BS $t^{\epsilon} \dot{e} k k \bar{i} \dot{m} \bar{a} k \cdot k a t \mid$

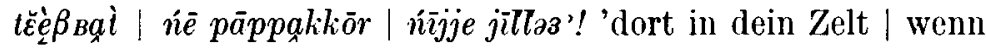
du ankommst, | deine jüngere Schwester | lebe nicht!' (366);

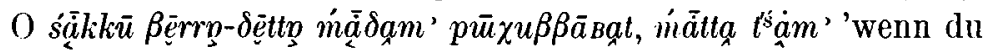
das Zelt des Gänseknöchelhaar-Wirtes suchst, sein Zelt ist dieses' (465).

Die Trennung der Verbindung der zwei Prädikate (und der 
Eräänzungen) wird begünstigt, wenn zwischen den Verbindungen ein Adverb erscheint (vgl. Károly, ebd. 131): 1 T padxr

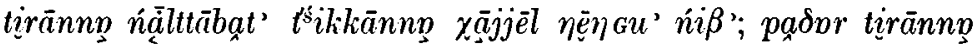

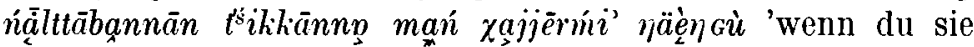
[die Lärche] mit der Faust durchbohrst, dann ist die Sonne dein, durchbohrst du sie nicht mit der Faust, aber wenn ich sie mit der Faust durchbohre, dann ist die Sonne mein' (91). Im folgenden ziemlich komplizierten Satz gehören zwei verbalnominale Prädikate zum verbalen Prädikat, das eine bezieht sich auf das Subjekt in der 1.P.Sg., das zweite auf das Subjekt in der 3.P.Pl, die einzelnen Verbindungen werden durch Ad-

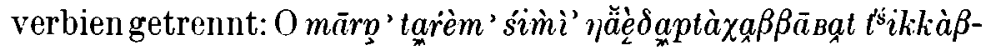

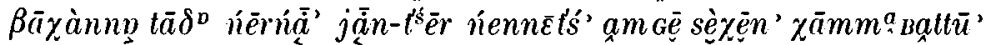

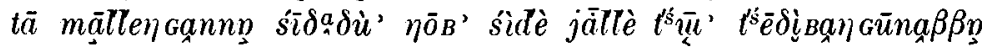
'gut, wenn du mich so schickst, denı wemn von jetzt an die auf der Erde wohnenden Menschen in irgendwelches Unglück geraten, rette ich sie dann in ein paar Tagen' (24).

Das Possessivsuffix des verbalnominalen Prädikats weist auf das Subjekt in der 2.P.Du. bzw. der 3.P.Pl. hin: O $t \bar{a} \delta^{v} p \bar{o} n^{\prime}$

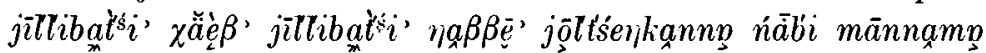
'dann ob sie lange oder kurze Zeit lebten, sagte irgendwann der

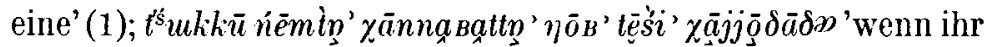
dieses Weib mitnehmt, bleibt ihr ohne Rentiere' (161). - Das verbalnominale Prädikat ist eine zweigliedrigeKonstruktion: das Verbalnomen von 'sein' mit dem Suffix * pa wird mit dem Verbalnomen auf $m m \bar{\imath}$ bzw. $\beta \beta \bar{\imath}$ verbunden: (C) wued tālei

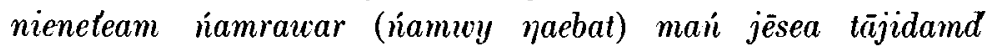
'hättest du den Dieb nur ergriffen, so hätte ich dir Geld ge-

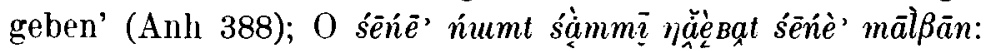

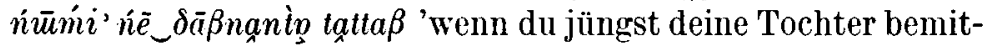
leidet hättest, hättest du eben gesagt, meine Tochter gebe ich als Austausch für die Frau' (444). - In Fragen erscheint das auf das Subjekt bezügliche Verbalnomen als alleiniges Prädi-

${ }^{1}$ Im Jurakischen gibt es keine Bindewörter, aber einige Adverbien,

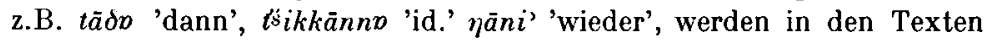
fast als kopulative Bindewörter gebraucht, ebenso wie z.B. in der ungarischen Volkssprache azután 'dann'. 


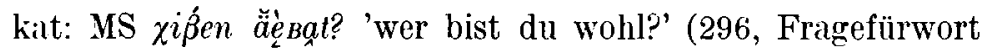

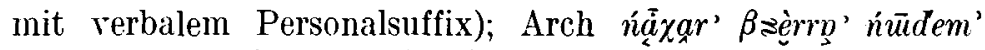

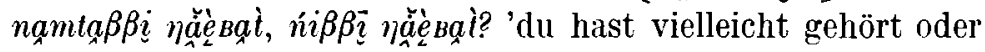
auch nicht von dem Jüngsten der Drei Bǒèrrn?' (438). - Auch die jurakische Sprache drückt die nachdrückliche Aufforderungr - wie die ungarische (vgl. Károly, ebd. 153, 188, 230) --

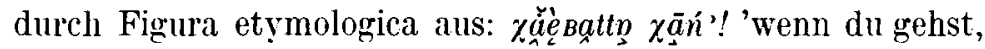
so gehe!' (Mat 443). Wenn in diesem Ausdruck -ttp < ${ }^{*} n+{ }^{*} t$, steht die jurakische Aufforderung auch der Konstruktion nach dem charakteristisch ungarischen Ausdruck jörén eljö 'veniens veniet' nahe. (Die beiden Glieder des jurakischen Ausdrucks stammen - wenn man die Lautwandel in Betracht zieht - von einem gemeinsamen Verbalstamm.) Ähnliche jurakische Ausdrücke: PD māmpattn mānnamng 'sagend sagte er' (Mat 406); Arch mämpattū'tarem' $m \bar{a}$ ' 'sagend trugen sie vor' (436). Das Kasussuffix $n$ erscheint in Verbindung mit einem Vokalelement in einer Figura etymologica: (C) mansiuràn ( mansiuäna) madm' 'ich, ich sagte [sagend (Opt.-Kond.) sige ichl' (128).

Das Verbalnomen auf ${ }^{*} p a$, das auf ein Subjekt in der 3.P. hinweist, erscheint in erzählenden Texten (selten auch in Zitaten) schon eigentlich als verbales Prädikat. In solchen Ausdrücken wird die Verbindung des verbalnominalen und des verbalen Prädikats und ihrer Ergänzungen in Form von zwei Gliedsätzen selbständig: das Abhängigkeitsverhältnis wird durch zwei koordinierte Sätze ausgedrückt. Die gleiche Endung der Prädikate und die besonderen Subjekte begünstigen die Verselbständigung der Verbindungen, das Subjekt des verbalen Prädikats ist oft aluch genannt: (C) udahad njamāda, | talnjā naekalpada 'ler| nahm ihn bei der Hand, | nach hier zieht er ihn'

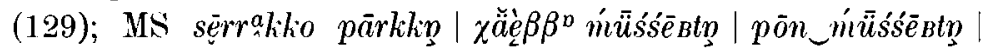

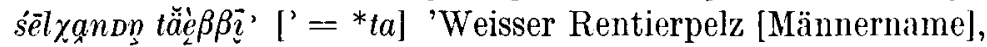
ob er kurze Zeit reiste, | ob er lange reiste, | traf bei seinem

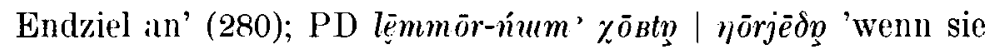
junge Sperlinge [Sr.] findet, | isst sie sie' (389); - (C) tarem jādabata ! lambita jāptsohana | hādada zjurā. | tarem sīrpata | niernda njāna | mar yadimeä 'Während er so wanderte, | am hinteren Ende seiner Skis | wirbelte der Schnee. | Als er blickte ! 
in der Richtung vor ihm | kam eine Stadt in Sicht' (290);

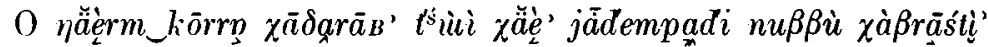
'unterliegt der Stier des Nordens, senden die oben befindlichen Gewittergeister warmes Wetter herab' (17). - Das auf das Subjekt in der 3.P. bezügliche verbalnominale Prädikat kann auch durch eine zweigliedrige Konstruktion ausgedrückt werden: (C) hübe tōbata (tōuy raebata), jiheradm 'ich weiss nicht, wer gekommen ist' (Anh 381). Die verbalnominale Konstruktion erscheint in Behauptungssätzen und Frage-

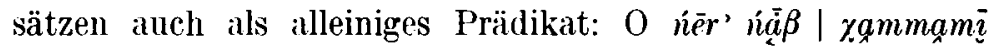

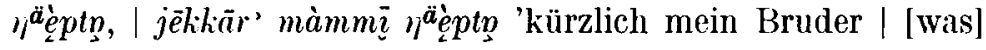
sagte er wohl, | ich weiss nicht, ob er sagte' (207); (C) tjäta titiliou, | nieven päpan'ah | seänja hunundo'oh | tōvui äeptōh 'meine vier Mutterbrüder, | die jüngeren Brüder meiner Mutter, | irgendwann | sind wohl gekommen' (281). - Die mit dem Possessivsuffix der 3.P. versehenen Formen des Verbalnomens kommen in der Sprache der Gesänge und auch in der gesprochenen Sprache als alleinige Prädikate vor: BM sa

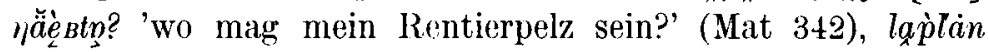

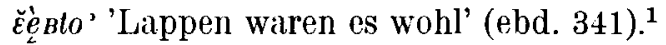

In der Sprache der ungarischen Kodexe fehlen Prädikate vom Typ *látcája, *liérvéje, die iuf ein Subjekt in der 3.P.Sg. hinweisen, gänzlich, im Jurakischen hingegen fehlen die als Prädikat fungierenden Verbalnomina auf *pa mit dem Possessivsuffix der 1.P.Ser. vom Typ látrám. ${ }^{2}$ In Verbindung mit einem

${ }^{1}$ Das Verbalnomen auf *pa ohne Personalsuffix kann im Satz ebenso eine Modalbestimmung sein wie das ungarische Verbaladverb auf 'a/ve in Ausdrücken vom Typ elbagyadva fekszik 'ermattet liegen'

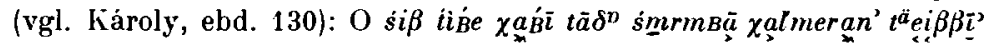
'der Siebenklafter Ostjake kam hierauf laufend zu dem Toten' (89) usw. Im Jurakischen wird das Verbalnomen, das ein determinierendes Possessivsuffix annimmt, auch als Attribut gebraucht: (C) njāmbada uঠado' | tün sibengäe | pülaraemd'die Hand, welche sie festhalten,

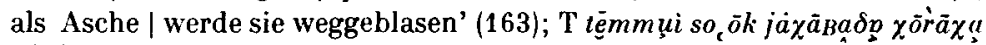

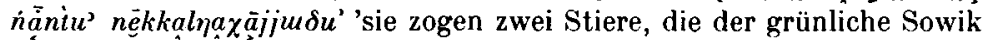
ab́gehäutet hatte, zu sich' (Mat 422).

${ }^{2}$ Auch solche Prädikate fehlen, die auf ein Subjekt der 1.P.Du. und

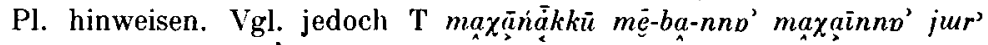

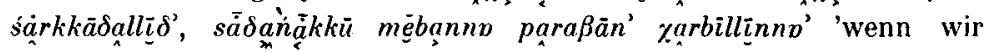


Subjekt in der 1.P.Sg. erscheint der Lativ des Verbalnomens auf ${ }^{*} p a$ bzw. ${ }^{*} p a+n a,{ }^{*} t a+p a+n a,{ }^{*} p a+t a+n a$ in prädikativer Funktion. An den Lativ wird kein Personalsuffix angehängt, in einigen Fällen bezeichnet das Personalpronomen mań 'ich' das Subjekt.'

Alle hierher gehörigen Belege sind Zitate aus Gesprächen von Personen. In einem solchen Satz wie BS jĭltēsan tūttām, 'wenn ich lebe, komme ich' (323) fällt die adverbiale Funktion mit der prädikativischen zusammen. Die zwei Funktionen trennen sich, wenn der Lativ und das verbale Prädikat Ergänzungen haben. Und noch deutlicher ist die prädikative Funktion des Lativs dann, wenn das durch den Lativ ausgedrückte Geschehen und das verbale Prädikat kein gemeinsames Subjekt haben: (C) anina töban | tjikēnja sium idanjin 'wenn ich ein anderes Mal knmme, I dann hängst du mich wohl' $(92)$; jilëbnān ( jilieb$\left.n^{\prime(i n}{ }^{2}\right) \mid$ sit'ch hōptsum, häbnan | tjiki jāna | sēbemsi beanor! 'wenn ich lebe, finde ich dich, wenn ich sterbe, an dieser Stelle beginne zu verfaulen!' (79). Aus der O-Mundart kann ich mehrero

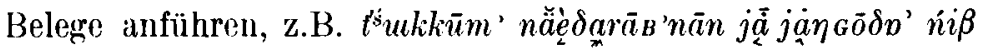
'wenn ich diese loslasse, ist ja keine Erde vorhanden' (3);

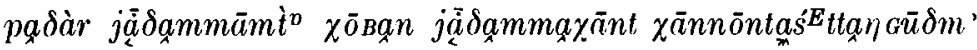
'wenn ich deine Spuren treffe, werde ich deinen Spuren opfern

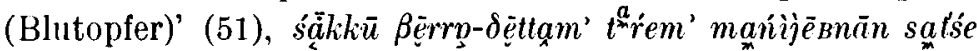
$j \bar{a} \dot{a} \dot{b} \imath$ lji' 'Gänseknöchelhaar-Wirt wie ich ihn so sah, wurde sehr betrunken' (455, in einer Variante des Satzes: mañìjēBnañi' 451). - In Fragesätzen erscheint der Lativ des Verbalnomens als

mit dem Rücken dagegen stehen, zischte das Fett unserer Rücken, wenn wir mit dem Gesicht darauf zu sind, sind wir am Verbrennen' (55; me-'sein'). In einer Frage ist das Verbalnomen mit dem Pos-

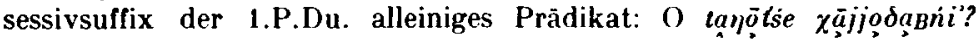
'bleiben wir zu übersommern?' (444).

1 Jach vereinzelten Belegen nimmt der Lativ das Possessivsuffix

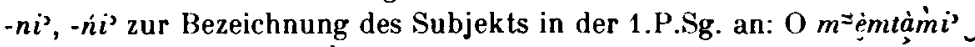

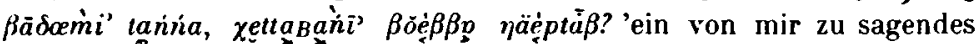
Wort ist vorhanden, ob es wohl schlimm ist, wenn ich erzãhle?' (28);

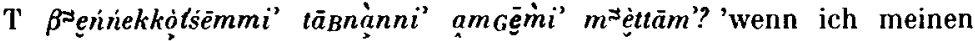
Hund gebe, was werde ich haben?' (87).

${ }^{2}$ Im Beleg jiliebnäm auf Seite 178 der FLF XXXVIII ist das $m$ am Wortende ein Schreibfehler, ein Irrtum. 
alleiniges Prädikat: (C) hüvium miritabta, | mūng miritabta |harn miritaban? 'wer von beiden ist schneller, | ist der Pfeil schnel-

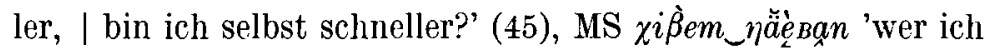

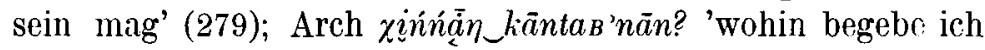
mich?' (423).

Es darf nicht ausser acht gelassen werden, dass der Lativ nach einigen Belegen - mit den entsprechenden Possessivsuffixen versehen - auch in Verbindung mit Subjekten in der 2. und 3. Person in prädikativer Funktion vorkommt: (C) seänakōsi jiherābnand | amgam saedorān? 'wenn du nicht spielen kannst, | was nähst du?' (197); BS pā $\delta a \grave{\beta} \grave{\imath}$ laptäąeikkom' |

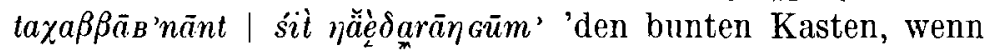
du ihn gibst, lasse ich dich los' (203); (C) num tónam tābnanda, mań yaewa mirim mirtejidamd 'gibt Gott Füchse, so werde ich meine Kopfsteuer bezahlen' (Anh 382); MS to'oprinnn msès'-

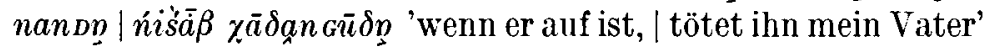
(346). - Das verbale Prädikat steht manchmal mit seinen Ergänzungen vor der Verbindung des durch den Lativ ausge-



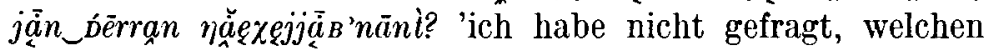
Landes Bewohner du sein magst?' (438). In einer Frage kann der Lativ des Verbs 'sein' auch als alleiniges Prädikat erscheinen: (C) amgae äebnanda? 'was mag es sein?' (7); amgae äebnāndi'? 'was mögen sie [Du.] sein?' (14); amgae äebnandoh? 'was mögen sie sein?' (77). Auch in der gesprochenen Sprache:

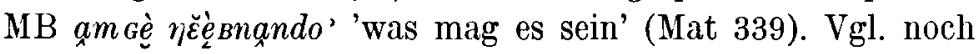

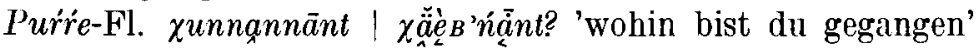
(486, Zaubergesang). ${ }^{1}$

Wir können es für wahrscheinlich halten, dass der prädikative

IIm Jurakischen kommt das Verbalnomen auf ${ }^{*} p \varepsilon$ in prädikativer Funktion nicht vor. Von den mit ${ }^{*} p \varepsilon$ gebildeten Verbalnomina nominalen Charakters kann (Wb 128) jil ese, jillebe 'Reichtum (an Rentieren, an Geld)' erwähnt werden, das in den Texten hauptsächlich in der Bedeutung 'Rentierherde' vorkommt. Das Wort stammt aus dem Verbalnomenstamm jillle- $\left(<^{*} j \bar{l} l+j \varepsilon\right)$, und seine Bedeutung entspricht der volkssprachlichen Bedeutung 'Getreide' des ungarischen

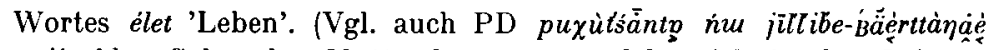
$\chi_{2}^{a j j e}$ 'der Sohn des Mütterchens verwandelte sich in den Lebens(mittel)spender-Geist' 132). In den Texten findet sich das Wort auch in 
Gebrauch der personalsuffigierten Formen und des Lativs des jurakischen Verbalnomens auf ${ }^{*} p a$ eigentlich nur als Eigenart der traditionellen Texte vorhanden ist. In den Texten, aus denen wir das Leben der Juraksamojeden kennenlernen können (Mat 339-373), sind mir mit Ausnahme einiger Fragesätze nur

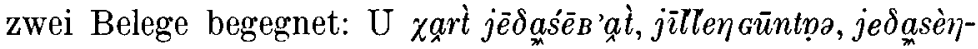

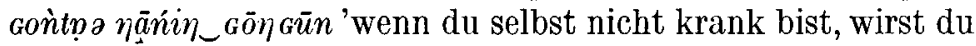
leben, ohne Krankheit wirst du sie [die Lebensmittel] wieder

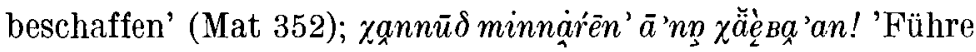
den Schlitten, wenn ich fortgehe' (ebd. 364). Obwohl sich in der Sprache der ungarischen Kodexe ein viel differenzierterer Sprachzustand spiegelt als in den traditionellen jurakischen Texten, ist es doch unzweifelhaft, dass zwischen dem prädikativen Gebrauch der ungarischen Verbaladverbien auf va/ve und ván/vén und dem des jurakischen Verbalnomens auf *pa und dem Lativ des Verbalnomens ein enger und urtümlicher Zusammenhang besteht. Wie dieser Zusammenhang zu erklären ist, diese Frage lässt sich zur Zeit nicht beantworten. Es ist die Aufgabe der Forschung in der Zukunft, die morphologischen und syntaktischen Gemeinsamkeiten der samojedischen und der ugrischen Sprachen aufzudecken und ihre urgeschichtlichen Hintergründe - soweit möglich - zu beleuchten. Was die Methode der Lösung der Probleme betrifft, so ist es gewiss, dass Konzeptionen und Theorien, die nicht durch sprachliche Tatsachen untermauert sind, zu keinen positiven Ergebnissen führen können. Die offenen Fragen kann man erst nach systematischer Untersuchung des sprachlichen Materials, nach viel Zeit, Mühe und Geduld kostenden Einzeluntersuchungen und auf Grund historischer Anschauungsweise beantworten.

IRENE N.-SEBESTYÉN

der Bedeutung 'Leben': (C) manj jiheram | jilebeän ( jilimian) jamban | seanakosi jiheram 'ich kann nicht mein Leben lang | verstand ich nicht zu spielen' (196); T jilltebe sérmńe mań jă $\bar{a} \delta e r \eta \bar{a} \delta^{n} m$ ' 'in Lebensangelegenheiten wandere ich' (53).

\section{ABKÜRZUNGEN}

$\mathrm{C}+\mathrm{Anh}=\mathrm{M}$. Alexander Castréns Wörterverzeichnisse aus den samojedischen Sprachen. Anhang. Materialien zu einer Syntax. Bearbeitet von Anton Schiefner. St. Petersburg 1855. 
$\mathrm{C}+$ Seitenzahl $=$ Samojedische Volksdichtung. Gesammelt von M. A. Castrén. Herausgegeben von T. Lehtisalo. MSFOu LXXXIII. Helsinki 1940.

$\mathrm{Wb}=\mathrm{T}$. Lehtisalo, Juraksamojedisches Wörterbuch. Helsinki 1956. Seitenzahl = Juraksamojedische Volksdichtung. Gesammelt und herausgegeben von T. Lehtisalo. MSFOu XC. Helsinki 1947.

Mat $=$ Samojedische Sprachmaterialien. Gesammelt von M. A. Castrén und T. Lehtisalo. Herausgegeben von T. Lehtisalo. MSFOu 122. Helsinki 1960. 